\title{
Generation of electric currents via neutral-ion drag in the chromosphere and ionosphere
}

\author{
V. Krasnoselskikh*, W.P. Abbett ${ }^{\dagger}$, H. Hudson ${ }^{\dagger}$, G. Vekstein** and S.D. \\ Bale $^{\dagger}$
}

\author{
${ }^{*}$ LPC2E, CNRS-University of Orléans, 3A Avenue de la Recherche Scientifique \\ 45071 Orléans CEDEX 2 FRANCE \\ ${ }^{\dagger}$ SSL, UC Berkeley, CA, USA 94720 \\ ${ }^{* *}$ School of Physics and Astronomy, The University of Manchester, \\ Alan Turing Building, Manchester M13 9PL UK
}

\begin{abstract}
We consider the generation of electric currents in the solar chromosphere. The ionization level in this region is generally supposed to be low. We show that the ambient electrons are magnetized even for weak magnetic fields $(30 \mathrm{G})$, i.e. their gyrofrequency is larger than the collision frequency; ion motions continue to be dominated by ion-neutral collisions in this region. Under such conditions the ions are dragged by neutrals. As a result, the dynamics of magnetic field resembles frozen-in motion of the field with the neutral gas. On the other hand magnetized electrons drift under the action of the electric and magnetic fields induced in the reference frame of ions moving with the neutral gas. This relative motion of electrons and ions results in the generation of quite intense electric currents. The dissipation of these currents leads to the resistive electron heating and efficient gas ionization. Ionization by electron-neutral impact does not alter the dynamics of the heavy particles; thus the gas turbulent motions persist even when the plasma becomes fully ionized and the resistive current dissipation continues to heat electrons and ions. This heating process is so efficient that it can result in typical temperature increases with altitude as large as $0.1-0.3 \mathrm{eV} / \mathrm{km}$. We conclude that this process can play a major role in the heating of the chromosphere and corona. We show that the physical conditions in the solar chromosphere, in particular the neutral and ion density dependencies upon altitude, are very similar to those in the lower ionosphere of the Earth. A very similar process of current generation occurs in the ionosphere after strong earthquakes, resulting in the generation of strong perturbations in the ionosphere. We then present well-known results of the observations of such perturbations, which allow an evaluation of the increment of the growth of the perturbations with altitude, making use of ionospheric sounding. These results are in perfect agreement with estimates obtained making use a model similar to ours. We consider that these observations clearly show the efficiency of the physical mechanisms discussed, and thus provide strong support for our ideas.
\end{abstract}

Keywords: Chromospheric heating, Ionospheric perturbations after earthquakes

PACS: $96.60 . \mathrm{Na}$, 52.25.Jm, 94.20.Bb

\section{INTRODUCTION}

The detailed physical mechanism of coronal heating is not yet well understood. A number of fundamental questions remain that challenge theoretical descriptions and the interpretation of observational data (see, e.g. Klimchuk [1], Walsh and Ireland [2], for recent reviews). In addition, the heating of the chromosphere requires much more energy than the heating of the corona, and this related process is also difficult to understand.

Parker [3] proposed the idea that the solar corona could be heated by the episodic dissipation of energy at many small-scale tangential discontinuities arising spontaneously 
in the coronal magnetic field, as it becomes braided and twisted by random photospheric footpoint motions. These events - sudden changes of the magnetic field topology - hypothetically result in plasma heating and the acceleration of non-thermal particles. Parker invented a special name for these elementary energy release events, nanoflares. The inspiration for this was the discovery of the hard X-ray microflares [4]; the energy of one nanoflare was to be roughly $10^{-9}$ times the energy of a major flare, and thus orders of magnitude weaker than even the microflares. Parker's idea stimulated intensive searches for any observational signatures of nanoflares $[5,6,7]$ and their possible contribution to the overall energy budget of the solar corona [1].

Microflares were first detected in hard X-rays in a balloon-borne experiment [4]. The subsequent development of new instrumentation produced multi-wavelength satellite and ground-based high-resolution observations of smaller-scale $\left(\sim 10^{3} \mathrm{~km}\right.$ or even smaller) and lower-energy phenomena. Soft X-ray imaging revealed abundant microflares in active regions [8], and RHESSI observations found that virtually all of a sample of some 25,000 hard X-ray microflares occurred in active regions. Krucker et al. [9] found flare-like brightenings in areas of the quiet Sun, and observations at EUV wavelengths (e.g., the "blinkers" [10]) reveal bursting activity above the boundaries of the magnetic network. Similar phenomena that form small X-ray jets at the limb were reported by Koutchmy et al. [11]. From these and other EUV observations [12, 13], if not the hard X-rays, we conclude that Parker's idea of episodic heating of the apparently steady quiet corona should not be discarded, even though no convincing evidence for the required steepening [14] of the energy distribution function has yet been presented.

However, the idea of coronal heating via tangential discontinuities that arise spontaneously (nanoflares) does not address two important questions. Namely, where does the excess magnetic energy come from, and what was its original source? Parker's analysis considered ideal MHD statistical equilibria that contain multiple discontinuities. Later Rappazzo et al. [15] showed that a large-scale MHD energy source perturbed by slow motions on its boundary, supposed to be induced from the photosphere, results in the generation of a Poynting flux. This drives an anisotropic turbulent cascade dominated by magnetic energy. The result looks similar to Parker's tangling of magnetic field lines but the small-scale current sheets (which replace the tangential discontinuities) are continuously formed and dissipated. In this modification of the initial scenario the current sheets are the result of the turbulent cascade. The initial energy reservoir in such a view is contained in large scale magnetic-field structures.

Here, we discuss another possibility: the direct generation of relatively small-scale electric currents by neutral gas motions. Clearly, a huge energy reservoir exists in the form of turbulent motion of neutral gas at and beneath the photosphere, supported by the underlying convection zone. It is widely accepted that this energy can be partially transformed into excess magnetic energy in the chromosphere and corona. However, there is no quantitative model that describes the physical mechanism of the necessary energy transfer. The development of a model that describes this process from quasineutral gas motions to the magnetic field in a step-by-step manner inevitably raises questions about the spatial and temporal scales at which such a transfer can occur and about its location. Recent observations provide strong indications that the energy reservoir is indeed the dynamic turbulent photosphere, and that the energy transfer from the turbulent gas motions to various kinds of trapped and transient magnetic field 
oscillations takes place at the chromospheric level.

Analysis of the high resolution spatial ( $\sim 150 \mathrm{~km}$ at the Sun) and temporal (few s) data obtained by the SOT (the Solar Optical Telescope) aboard Hinode revealed that the chromosphere is dominated by a multitude of thin $(\sim 200 \mathrm{~km}$ wide) dynamic, jetlike "type II" spicules [16]. These are ejected upwards with characteristic velocities of $20-150 \mathrm{~km} / \mathrm{s}$ and reach heights of $2000-10000 \mathrm{~km}$ before disappearing from the chromospheric passband (in this case, the $\mathrm{H}$ line of $\mathrm{Ca}$ II). The type II spicules have short lifetimes $(10-300 \mathrm{~s}$; most of them last less than $100 \mathrm{~s})$ and many of them undergo substantial transverse displacements of the order of $500-1000 \mathrm{~km}$. Moreover, the largescale long-living spicules display oscillatory motions in the direction perpendicular to their own axes. Since the spicule structure can be taken to outline the direction of the magnetic field, this led the authors to the conclusion that the observed motions indicate Alfvénic perturbations.

Furthermore, the spatio-temporal variations of the chromosphere have always revealed the greatest complexity, and prominences also consist of numerous threadlike features with strong and mixed flows along these threads [17]. Observations with SOT have provided an exceedingly variable and dynamic picture of these flows and field structures [18]. The SOT chromospheric data are in the $\mathrm{H}$-line (Ca II), showing plasma at roughly $2 \cdot 10^{4} \mathrm{~K}$. The movie presented by Okamoto et al. (available on the Hinode web site http: //solarb.msfc.nasa.gov/) shows ubiquitous continuous motions along the prominence thread lines. The oscillatory motions observed might be interpreted in terms of propagating or standing Alfvén waves on the magnetic field that presumably structures the prominence. The typical transverse spatial scale of threads was found to be of the order of $600 \mathrm{~km}$, with a characteristic length of the order of several Mm. The characteristic temporal scale was found to vary from 100 to several hundred seconds.

SOT observations near the limb led to the discovery of another small-scale dynamic phenomenon in the chromosphere: tiny chromospheric "anemone jets," named for the similar X-ray features [19]. These jets resemble larger-scale features well-known in $\mathrm{H} \alpha$ data and termed "surges" or "sprays"; these often occur near sunspots and in association with flares or other transient activity (e.g. [20]). All of these observations can be considered as indications of the generation of small-scale perturbations and oscillations of the magnetic field in the chromosphere, where the degree of ionization is still relatively small.

Very similar problems have also been addressed via computer simulations. Several types of codes have been developed to study the dynamic, magnetic, and energetic connections between the convectively unstable layers below the visible surface of the Sun, and the overlying solar corona. To achieve this objective the various authors have developed numerical descriptions of the different physical processes, such as energy exchange and radiative transfer, as realistically as possible; they then combine these algorithms with an MHD description of the plasma/gas motions. The simulations aim to describe the evolving convection zone and corona within a single computational volume. A series of simulations of the quiet Sun in a domain that encompasses both the upper convection zone and low corona were performed making use of one of such codes by Abbett [21, 22]. These simulations use the so-called RADMHD code, which employs a unique combination of existing, well-studied algorithms to solve the MHD system semiimplicitly on a domain-decomposed computational grid. It allows one to address much 
of the inherent spatial and temporal disparity of the system. It is described by Abbett et al. $[21,22]$ where the results of the simulations are presented. Can the magnetic field generated by a convective surface dynamo account for some of the observed properties of the quiet-Sun atmosphere? This work disclosed that

- It is possible to heat a model corona to X-ray-emitting temperatures with the magnetic fields generated from a convective dynamo, and an empirically based heating mechanism consistent with the observed relationship between X-ray emission and magnetic flux observed at the visible surface;

- Within the limitations of the numerical models of the quiet Sun, the resistive and viscous dissipation alone are insufficient to maintain a hot corona;

- The quiet-Sun model chromosphere is a dynamic, non-force-free layer that exhibits a temperature reversal in the convective pattern in the relatively low-density layers above the photosphere;

- The majority of the unsigned magnetic flux lies below the model photosphere in the convectively unstable portion of the domain;

- Horizontally-directed magnetic structures thread the low atmosphere, often connecting relatively distant concentrations of magnetic flux observed at the surface; and

- Low-resolution photospheric magnetograms can significantly underestimate the amount of unsigned magnetic flux threading the quiet-Sun photosphere.

The results of these calculations $[21,22]$ that we present here were obtained making use of a box with $(x, y, z)$ dimensions of $2.6 \cdot 10^{5} \mathrm{~km}$ by $1.3 \cdot 10^{5} \mathrm{~km}$ by $2.6 \cdot 10^{5}$ $\mathrm{km}$, using $141 \cdot 111 \cdot 111$ grid points on a non-uniform mesh. The mesh points were concentrated near the lower boundary $D_{z}=290 \mathrm{~km}$ near $z=0$ (the solar surface), and $D_{x}=D_{y}=370 \mathrm{~km}$. A uniform resistivity $\eta$ has been used, corresponding to a Lundquist number $S=\tau_{R} / \tau_{A}=10^{4}$. Here, $\tau_{R}=\frac{4 \pi L^{2}}{\eta c^{2}}$ is the resistive diffusion time, and $\tau_{A}=L / V_{A}$ is the Alfvén time for a length scale $L=65000 \mathrm{~km}$. This is approximately the separation of the two poles of the emerging flux tube (after it has fully emerged). A uniform viscosity $v$ is also used, corresponding to $\tau_{v} / \tau_{A}=150$, where $\tau_{v}=L^{2} / v$ is the viscous diffusion time. The higher viscosity (relative to the resistivity) was used when relaxing a configuration toward a force-free state. One of the important questions that arose from the results of these studies, but not explicitly treated in previous publications, is related to the extremely strong electric fields that are generated as the result of turbulent motions. Indeed, the motions of plasma with characteristic velocities of several $\mathrm{km} / \mathrm{s}$ across the magnetic fields of the order of tens of Gauss result in the electric fields reaching several tens of $\mathrm{V} / \mathrm{m}$ and even more. We present here a description of the spatial distribution of velocity, magnetic and electric fields that are placed on the surface at an altitude of $580 \mathrm{~km}$ above the temperature minimum in the chromosphere.

Figures 1 represent the typical spatial distribution of the $y$ component of the magnetic field, at one moment of time during the run, and the spatial distribution of the $x$-component of the velocity. Figure 2 shows the spatial distribution of the $z$ and $x$ components of the electric field. One can see that the electric field can reach extremely high values, up to several hundred V/m. Figure 3 represents the characteristic correla- 
tion function of this field. Note also that the characteristic scale of the field variations is of order of $300 \mathrm{~km}$. The problem of current variations on such scales can lead to very important additional effects that are not included in such models. Taking into account the exponential decrease of the gas and plasma densities and the electron-ion-neutral collision frequency, one can suppose that locally the effects of the electric field can result in generation of the strong currents. From an entirely different point of view, an estimate of the electric field inside quiet coronal loops results in values of the order of $10^{-10}-10^{-9} \mathrm{~V} / \mathrm{m}$. Such small electric fields correspond to small potential differences between the feet of a loop of size $60000 \mathrm{~km}$, only of order of $1-10 \mathrm{~V}$. This poses questions: where are the strong fields located, and why don't they penetrate to high altitudes?
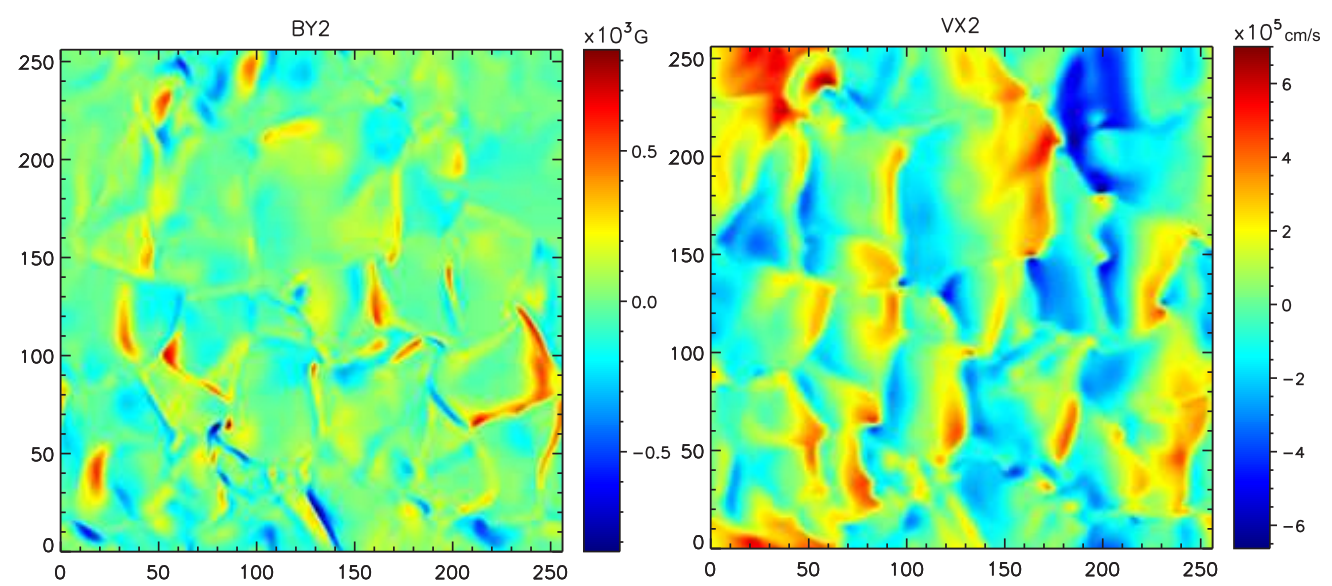

FIGURE 1. Left: Y-component magnetic field variations characteristic of a quiet-Sun model along a horizontal slice through the $30 \times 30 \times 7.5 \mathrm{Mm}^{3}$ domain at $580 \mathrm{~km}$ above the surface of the photosphere. Right: X-component of the velocity distribution for the same run along the same horizontal slice.
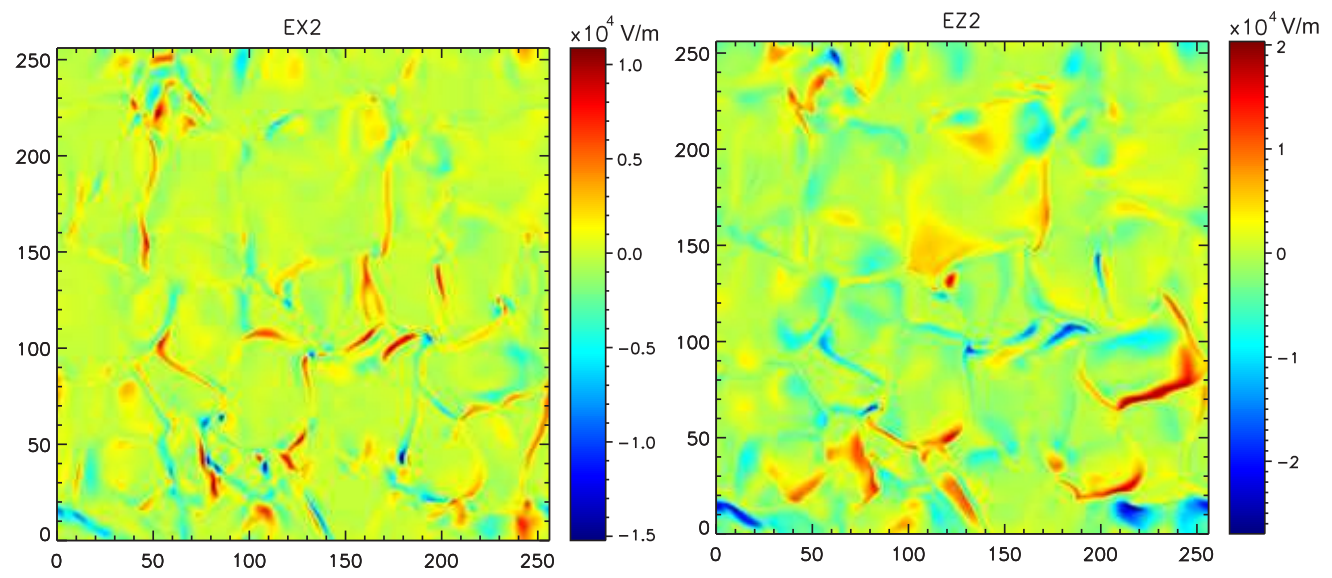

FIGURE 2. X (left) and $\mathrm{Y}$ (right) components of the electric field distributions for the same run along the same horizontal slice. 


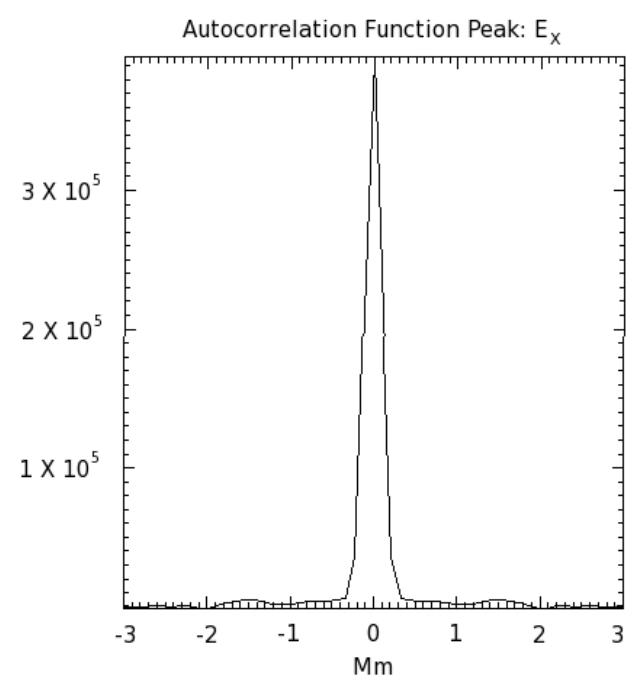

FIGURE 3. Spatial autocorrelation of the X-component of the electric field for the same run along the same horizontal slice averaged over Y-direction.

\section{THE COUPLING PROBLEM}

In spite of greatly improved observational data, the physics of chromospheric and coronal heating is not yet well understood. While the underlying mechanism must be associated with the magnetic field, the details of how efficiently the energy of convective motion is transformed into magnetic energy in the solar atmosphere is an open question. The importance of this process should not be understated. It is relevant not only to coronal heating and the acceleration of the solar wind, but also to the formation of the initial spectrum of wave turbulence introduced into the solar wind.

We propose a mechanism to generate electric current based on the Alfvén or magnetosonic waves that result from strong ion-neutral drag. We assume the photospheric motions to be turbulent, and to consist of both compressional and rotational flows with energies exceeding that which is necessary to drive the observed oscillations of suspended threads or jets in the chromosphere. The question is then how these motions are transported from the photosphere to the chromosphere, and how the flow of neutrals leads to motions of the charged particles and, ultimately, to the generation of electric current.

There are two physical processes that are necessary to convert the energy of the neutral gas motion into the magnetic field oscillations. Photospheric motions of charged particles are dominated by frequent electron-neutral and ion-neutral collisions, so that ions and electrons tend to follow the neutral gas motion with the zero net electric current.

Since density falls off rapidly with height in the solar chromosphere, the electron collision frequency of neutrals and ions decreases to the point where it becomes smaller than the electron gyrofrequency. However, the ion-neutral collision frequency at this height still remains substantially larger than the ion gyrofrequency, since ion-neutral and electron-neutral collision rates are not substantially different (e.g., [23]). Therefore, 
the motions of ions and electrons differ. Electrons tend to move along the magnetic field lines and drift due to $\mathbf{E} \times \mathbf{B}$ in the transverse direction, while the ions continue to move together with the neutrals. This difference results in the generation of electric currents as established in the following.

To view this situation from a different perspective, if ions move together with neutrals (due to the strong drag) with some angle to the background magnetic field, an inductive electric field $\mathbf{v} \times \mathbf{B}$ appears in the reference frame of the plasma. This induced electric field inevitably generates electric currents that can be calculated using the plasma conductivity tensor. When electrons and ions are both demagnetized, this tensor reduces to the simple scalar conductivity, and the resulting current is very small. When electrons become magnetized, the motions of electrons and ions become decoupled, and the efficiency of the current generation is substantially higher.

Thus, we conclude that there is an efficient chromospheric dynamo operating in the layer between the level of electron magnetization and the height where either the degree of ionization becomes high (comparable with unity) or where the ions become magnetized. Forced oscillating currents are generated most effectively when the inductive electric field has characteristic frequencies and wavelengths close to the eigenmodes of the system, i.e. the MHD type waves in the magnetized weakly ionized plasma. At higher altitudes, where ions are also magnetized, the motion of ions and electrons can still differ. However, under these conditions, both components will mainly move along the magnetic field lines and perform drift motions across the field. Thus, the current is directed mainly along the magnetic field lines, while in the intermediate region it can be generated in an arbitrary direction.

In summary the problem of the current generation by the turbulent flow of photospheric neutral gas involves two separate steps. First, the energy and vorticity of the photospheric motions will be transported upwards to the level where the electron and ion motions become de-coupled. Second, one must derive a self-consistent system of equations describing the inductive electric field and the resulting deformation of the background magnetic field. Finally, using the obtained electric field and current density estimates we may discuss the efficiency of the electron heating due to collisional heating and other possible processes.

\section{ATMOSPHERIC MODELS}

We appeal to standard semi-empirical atmosphere models for approximate values of the physical parameters of the plasma in the photosphere and the chromosphere. Fontenla [24] provides a recent series of eight such models representing features such as the quiet Sun, faculae, and sunspots. Such models are based on macroscopic radiative transport theory and are adjusted to recreate solar spectroscopic observations. They do not represent the dynamics, nor the plasma physics, since they are static single-fluid models. Figure 4 compares collision frequencies from the Fontenla quiet-Sun model with the gyrofrequencies of electrons and ions. For this estimate we assume a constant magnetic field of $30 \mathrm{G}$ [e.g. 26, 27].

The Fontenla models cover a range of physical features in the solar atmosphere, and were originally intended for irradiance modeling. Here, we use these models as a guide 


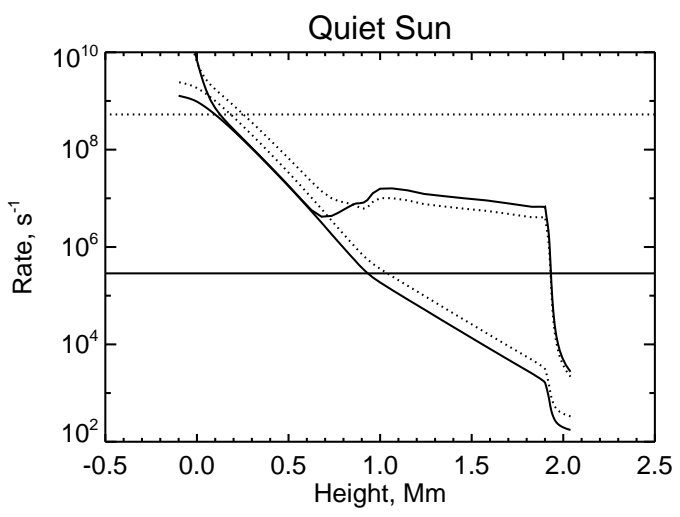

FIGURE 4. Collision frequencies in the quiet-Sun model of [24]. Solid (dotted) curves represent proton (electron) collision frequencies, with the lower lines showing neutral rates only [e.g. 23]; the horizontal lines show the gyrofrequencies (electrons and ions) for an assumed field strength of $30 \mathrm{G}$. Reproduced with permission, copyright (2010) Institute of Physics (IOP)

to set a range of parameters that are reasonably consistent - in this limited theoretical framework - with solar structure. Figure 5 shows the location of the chromospheric dynamo layer for the full range of models. The electrons are magnetized, for all models, essentially throughout the solar atmosphere. Thus the chromospheric dynamo layer begins close to the photosphere and extends high into the chromosphere. This is true of all of the Fontenla models, ranging from sunspot umbra to bright facula, for which the transition-region pressures range from 0.2 to about 2.0 dyne $\mathrm{cm}^{-2}$.

We emphasize that these models do not have any plasma physics in them as such, and only serve as references at the order-of-magnitude scale. Indeed, the mechanism discussed in this paper will certainly require drastic revisions of these models.

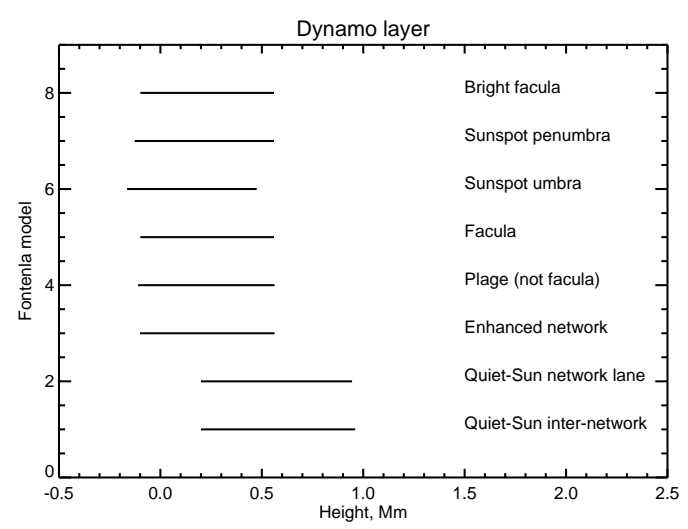

FIGURE 5. Chromospheric dynamo layers for the various solar features modelled by [24]. The field strengths assumed are $30 \mathrm{G}$ for the quiet Sun (the lower two models), and 1500G for the others. Reproduced with permission, copyright (2010) Institute of Physics (IOP) 


\section{ENERGY TRANSFER FROM THE PHOTOSPHERE TO CHROMOSPHERE}

We first consider the physics of mass, momentum, and energy transport between the photosphere and upper chromosphere. In order to formulate a mathematical description of the problem we need to take into account physical conditions at the level of the photosphere and chromosphere, as described above.

As shown by Krasnoselskikh et al. [28], the physics of mass, momentum, and energy transport between the photosphere and upper chromosphere can initially be described in the framework of the model of an isothermal atmosphere. The gas is supposed to have a constant sound speed and to be stratified by a uniform gravitational force acting in the negative z-direction, for which $\rho_{0}(z)=\rho_{p h} \exp (-z / H)$. Here $\rho_{p h}$ is the density at the level of the photosphere corresponding to $z=0, C_{S}^{2}=\left(\gamma P_{p h} / \rho_{p h}\right)$, is the adiabatic sound speed, $G$ is the gravitational acceleration and $\gamma$ the ratio of specific heats. The gas motions can be described by the equation of continuity, the equation of motion and the equation of state that for the sake of simplicity was chosen to be adiabatic. It was shown that solutions can be chosen in the form $P, \rho, v \sim \exp i\left(\omega t-k_{x} x-k_{y} y-k_{z} z\right)$, whence the problem can be reduced to the analysis of the dispersion relation that determines the dependency of the frequency $\omega$ upon components of the wave vector $\vec{k}$. The problem is formulated as a boundary-value problem where we assume the perturbations and their time dependences to be prescribed at the boundary $z=0$. The goal is to describe the dynamics of the perturbations at level $z=h$. In this case the very same equations should be used to find out the dependence of the component $k_{z}$ of the $k$-vector upon the frequency $\omega$ and other components $k_{x}, k_{y}$. Substituting the dependencies defined above into the linearized equations one can obtain the dispersion equation in the form

$$
\left.\left[\omega^{2}-i K g\right]\left\{\omega^{2}-C_{S}^{2}\left[\left(k^{2}+K^{2}\right)+\frac{i K}{H} \frac{\gamma-1}{\gamma}\right)\right]\right\}-i G C_{S}^{2}\left(k^{2}+K^{2}\right)\left(K+\frac{i}{H} \frac{\gamma-1}{\gamma}\right)=0,
$$

where $C_{S}^{2}=\gamma G H, k^{2}=k_{x}^{2}+k_{y}^{2}, K=k_{z}-\frac{i}{H}$ and

$$
k_{z}=\frac{i}{2 H} \pm \sqrt{\frac{\omega^{4}-\omega^{2} C_{S}^{2}\left(k^{2}+\frac{1}{4 H^{2}}\right)+(\gamma-1) k^{2} g^{2}}{\omega^{2} C_{S}^{2}}} .
$$

This analysis demonstrates that a wide class of perturbations satisfying the condition

$$
\omega^{4}-\omega^{2} C_{S}^{2}\left(k^{2}+\frac{1}{4 H^{2}}\right)+(\gamma-1) k^{2} G^{2}>0
$$

increase with height, and the characteristic growth rate is $\Gamma=m k_{z}=1 / 2 H$.

This phenomenon, the "effective growth" of the perturbations with altitude in a hydrostatic gas equilibrium with exponentially decreasing density is well known in the terrestrial atmosphere. It plays an important role in the propagation of infrasonic perturbations induced by explosions in the atmosphere [e.g. 29, 30]. The very same physical phenomena are supposed to play a very important role in the solar photosphere and chromosphere and were intensively studied in numerous computer simulations, 
including other important effects such as radiative transport [31]. Several results of these simulations add important features of neutral gas motions. It is worth mentioning that the authors note that the vorticity is mainly generated by baroclinic forces and tends to concentrate in tubelike structures the widths of which is comparable to the numerical resolution. Another important feature for the plasma heating consists in the formation of supersonic flows and shock-like structures. If shocks form systematically in these flows they could become an important factor of coronal heating. However according to Stein and Nordlund [31] the shocks are indeed observed at the edges of integranular lanes, but are a rare occurrence. At any one time, supersonic flow occurs in only $3-4 \%$ of the surface. The role of acoustic gravity waves is also intensively studied experimentally. Recently new experimental studies combining SOT/NFI and SOT/SP instruments operated on Hinode satellite and the Michelson Doppler Imager (MFDI) on $\mathrm{SOHO}$ with the $3 \mathrm{D}$ computer simulation were performed by Straus et al. [32]. The authors came to the conclusion that the gravity waves are the dominant phenomenon in the quiet middle/upper photosphere and that they transport sufficiently more mechanical energy than the high frequency $(>5 \mathrm{mHz})$ acoustic waves. In addition they conclude that the acoustic flux is $3-5$ times larger than the upper-limit estimate of Fossum and Carlsson [33]. These observations together with the numerical models allow one to consider that acoustic gravity waves are one of the most important energy sources; they can be considered as a reservoir of energy that might be transformed to magnetic field by the generation of electric currents.

One should notice here that the absolute magnitude of the density perturbation actually decreases with height, but it drops more slowly than the background density. This results in the growth of the relative density perturbation, written as $\rho / \rho_{0}$ in our notation. On the other hand the velocity perturbations actually grow exponentially. An important issue here is the characteristic vertical scale of this growth. Taking the gas temperature to be $5000 \mathrm{~K}$ we find $H=\frac{k_{B} T}{M G}=140 \mathrm{~km}$. This linear analysis includes only the effect of the growth of the velocity and relative density perturbations with the altitude, hence, the perturbation scales do not vary with altitude. Of course, there are a wide range of phenomena, such as vortical flows, that are not accounted for in this formalism.

For example, the vortex radius can decrease with altitude. Indeed, assuming conservation of angular momentum, the increase of the velocity should result in the shrinking of a the transverse diameter of the vortex. This effect is relevant for Rossby vortices in a multi-layer atmosphere and it is in a good agreement with the observation of Stein and Nordlund [31] that the vorticity concentrates in small scale tubes.

\section{MAGNETIC PERTURBATIONS PRODUCED BY THE TURBULENT MOTIONS OF THE NEUTRAL GAS}

Turbulent convective fluid motions at the photospheric level extend upwards into the lower chromosphere. The latter is a weakly ionized plasma with a typical temperature of $6000-7000 \mathrm{~K}$, neutral hydrogen density $n_{H} \sim 10^{12}-10^{14} \mathrm{~cm}^{-3}$, and electron density $n_{e}$ $\sim 10^{10}-10^{11} \mathrm{~cm}^{-3}$. Under these conditions the frequency of the ion-neutral collisions is as high as $v_{\text {in }} \approx 10^{7}-10^{9} s^{-1}$ which, as will be confirmed below, produces quite a strong drag effect resulting in the bulk velocity of ions, $V_{i}$ closely matching that of the 
neutral gas, $V_{n}$. Then the magnitude of the generated electric current $\vec{j}=n_{e} e\left(\vec{V}_{i}-\vec{V}_{e}\right) \approx$ $n_{e} e\left(\vec{V}_{n}-\vec{V}_{e}\right)$ is determined by the electron bulk velocity $V_{e}$. The latter is governed by the equation of motion for the electrons

$$
m_{e} \frac{d \vec{V}_{e}}{d t}=-e\left\{\vec{E}+\left[\vec{V}_{e} \times \vec{B}\right]\right\}-\left(v_{e n}+v_{e i}\right) m_{e}\left(\vec{V}_{e}-\vec{V}_{n}\right)
$$

where $v_{e n} \approx 5 \times 10^{8}-10^{10} s^{-1}$ is the frequency of electron-neutral collisions, the major source of the electron drag. $v_{e i}$ is electron-ion collision frequency.

Ions are supposed to be dragged by neutrals, thus the velocity of electrons reads $\overrightarrow{V_{e}}=\overrightarrow{V_{n}}-\vec{j} /$ en .

The physical processes we describe occur in a parameter range corresponding to the transition in electron motions from unmagnetized and collision-dominated, to magnetized, when initially $\left|\omega_{B e}\right|<v_{e n}+v_{e i}$, and then with the growth of altitude $\left|\omega_{B e}\right|$ becomes larger than $\left(v_{e n}+v_{e i}\right)$. We consider hereafter slow motions $\omega \ll\left(v_{e n}+v_{e i}\right),\left|\omega_{B e}\right|$ similar to those we described in the previous paragraph. In this case one neglect the electron inertia in the left-hand side of Eq. (1). Then it can be simplified to obtain

$$
-e\left\{\vec{E}+\left[\overrightarrow{V_{n}} \times \vec{B}\right]-\frac{1}{n e}[\vec{j} \times \vec{B}]\right\}+\left(v_{e n}+v_{e i}\right) m_{e} \frac{\vec{j}}{n e}=0 .
$$

Applying the curl operator and using $\partial_{t} \vec{B}=-\nabla \times \vec{E}$, one obtains the final equation

$$
\frac{\partial \vec{B}}{\partial t}=\nabla \times\left[\overrightarrow{V_{n}} \times \vec{B}\right]-\frac{1}{e \mu_{0}} \nabla \times \frac{1}{n}[\nabla \times \vec{B} \times \vec{B}]-\frac{\left(v_{e n}+v_{e i}\right) m_{e}}{e^{2} \mu_{0}} \nabla \times \frac{\nabla \times \vec{B}}{n},
$$

where $\vec{j}$ is replaced by $\vec{j}=\frac{1}{\mu_{0}} \nabla \times \vec{B}$.

One can assume that the chromospheric magnetic field can be represented as $\vec{B}=$ $\overrightarrow{B_{0}}+\vec{b}$, where $\overrightarrow{B_{0}}$ is a background field, which for the sake of simplicity is assumed here to be just a uniform field, while $\mathbf{b}$ is a relatively small field deformation caused by a prescribed flow of neutral gas with velocity $\vec{V}_{n}$ and frequency $\omega$. Then the linearized (with respect to b) version of Eq. (3) takes the form

$$
\vec{b}+i d_{e}^{2} \frac{\left|\omega_{B e}\right|}{\omega}(\vec{h} \cdot \nabla) \nabla \times \vec{b}-d_{e}^{2}\left(1+i \frac{v_{e n}}{\omega}\right) \nabla^{2} \vec{b}=i \frac{B_{0}}{\omega}\left\{(\vec{h} \cdot \nabla) \overrightarrow{V_{n}}-\vec{h}\left(\nabla \cdot \overrightarrow{V_{n}}\right)\right\}
$$

where $\mathbf{h}$ is the unit vector along the background magnetic field, and $d_{e}=c / \omega_{p} e$ is the skin depth.

Let us now estimate the relative roles of the different terms in the left-hand side of Eq. (4). For $n_{e}=10^{11} \mathrm{~cm}^{-3}$ the electron plasma frequency is $\omega_{p e}=10^{10} \mathrm{~s}^{-1}$, which yields the $d_{e} \approx 3 \mathrm{~cm}$. The typical frequency of the neutral gas flows under consideration is $\omega \approx\left(10^{-2}-10^{-3}\right) \mathrm{s}^{-1}$ and their length scale is $L \sim 10^{2}-10^{3} \mathrm{~km}$. For $B_{0} \approx 100 \mathrm{G}$ the electron gyrofrequency $\omega_{B e}=2 \times 10^{9} \mathrm{~s}^{-1}$, while the characteristic electron-neutral collision frequency $v_{e n} \sim\left(10^{8}-10^{9}\right) \mathrm{s}^{-1}$. Therefore the ratio of the second and third terms compared to the first can be considered small. Thus Eq. (4) can be reduced to

$$
\vec{b} \approx i \frac{B_{0}}{\omega}\left\{(\vec{h} \cdot \nabla) \overrightarrow{V_{n}}-\vec{h}\left(\nabla \cdot \overrightarrow{V_{n}}\right)\right\}
$$


which means that in the lower chromosphere the magnetic field is effectively frozen into the neutral-gas flow.

This describes the magnetic field generation due to the motions of conductive fluid, neglecting small-scale effects scaling as the electron inertial length, and assuming the motions to be slow: $\omega \ll \Omega_{e}$. Let us now verify that the assumption made above, namely that the bulk velocity of the ions $\vec{V}_{i}$ is so close to that of the neutrals $\overrightarrow{V_{n}}$ that the electric current can be written as $\vec{j}=n_{e} e\left(\vec{V}_{n}-\vec{V}_{e}\right)$. It can be easily found from (5) that

$$
\vec{j}=\frac{1}{\mu_{0}} \nabla \times \vec{b}=i \frac{B_{0}}{\mu_{0} \omega} \nabla \times\left\{(\vec{h} \cdot \nabla) \overrightarrow{V_{n}}-\vec{h}\left(\nabla \cdot \overrightarrow{V_{n}}\right)\right\} \approx i \frac{B_{0}}{\mu_{0} \omega}(\vec{h} \cdot \nabla) \nabla \times \overrightarrow{V_{n}} .
$$

Using Eq. (5), the electric current can be estimated as $j \sim \frac{b}{\mu_{0} L} \sim \frac{B_{0} V_{n}}{\mu_{0} \omega H L}$. The velocity of the current -carrying electrons is

$$
\delta v_{e} \sim \frac{j}{n e} \sim \frac{e B_{0} V_{n}}{\mu_{0} m \omega H L} \frac{m}{n e^{2}} \sim \frac{\omega_{B e}}{\omega} \frac{d_{e}^{2}}{H L} V_{n}<V_{n} .
$$

A simple estimate of the currents generated at different altitudes and for different values of the magnetic field can be obtained by assuming the neutral gas motions to be rotational. In this case, the characteristic velocity in the vortex $V_{n}$ of characteristic spatial scale $L$ rotating with the characteristic frequency $\omega$ can be evaluated as $V_{n} \sim \omega L$. Thus, the characteristic magnetic field perturbation is $\delta B \sim B_{0} L / H$, and consequently the current density is $j \sim \delta B / \mu_{0} L \sim B_{0} / \mu_{0} H$. One can see that the magnetic field perturbations can become quite large; the current densities for magnetic fields of the order of $100 \mathrm{G}$ can become as great as $j \sim B_{0} / \mu_{0} H \sim 10^{-2}-10^{-1} \mathrm{Am}^{-2}$.

It is worth noting that this mechanism is dependent upon the velocity shear. An interesting feature of this process is the very high efficiency of the field generation around the boundaries of the neighbouring vortices, where the characteristic shear of the velocity of the gas motions is large. In this case the characteristic velocity shear is $V_{n} / L \gg \omega$; thus smaller-scale magnetic-field structures can be generated quite efficiently and magnetic fields generated and current densities can become tens or hundreds of times higher than the estimate above.

\section{ELECTRON AND ION RESISTIVE HEATING}

To evaluate the electron heating efficiency, one should find the induced electric fields parallel and perpendicular to the magnetic field. This can be done by using the component of the equation of motion for electrons along the magnetic field (Eq. 1).

$$
m_{e} \frac{d V_{e \|}}{d t}=-e E_{\|}-\frac{v_{e n} m_{e}}{n e} j_{\|}
$$

in the lowest-order approximation one should take into account that the frequency $\omega$ of wave motions is much smaller than the electron neutral collision requency. This leads to a classically collisional resistivity along the magnetic field, where the dominant effect is 
due to electron-neutral collisions

$$
\begin{gathered}
E_{\|}=-\frac{v_{e n} m_{e}}{n e^{2}} j_{\|}=-\frac{v_{e n}}{\varepsilon_{0} \omega_{p}^{2}} j_{\|}= \\
-\frac{i v_{e n}}{\mu_{0} \varepsilon_{0} \omega_{p}^{2}} \frac{B_{0}}{\omega}\left(\vec{h} \cdot \nabla \times\left\{(\vec{h} \cdot \nabla) \vec{V}_{n}-\vec{h}\left(\nabla \cdot \overrightarrow{V_{n}}\right)\right\}\right) \sim v_{e n} \frac{d^{2}}{L} \delta B .
\end{gathered}
$$

To evaluate the parallel electric field one should take into account the decrease of the electron-neutral collision frequency with height, from $v_{e n} \sim 5 \times 10^{8}$ to $10^{10}$, and the plasma frequency varies in its turn from $10^{10}$ to $10^{11}$. Taking the characteristic current densities obtained above we find electric fields in the range $E_{\|} \approx 10^{-2}-10^{-1} \mathrm{~V} / \mathrm{m}$, with the electron heating written [34]

$$
\frac{3}{2} N_{e} \frac{d_{e} T_{e}}{d t}+p_{e} \nabla \cdot \overrightarrow{V_{e}}=-\nabla \cdot \overrightarrow{q_{e}}+Q_{e}
$$

Here $p_{e}$ is the electron pressure, and the electron heating $Q_{e}$ is determined by two "friction forces," one due to the relative velocity between electrons and ions/neutrals $\left(\overrightarrow{R_{e i}}, \overrightarrow{R_{e n}}\right)$, and another due to the electron temperature gradient $\overrightarrow{R_{T}}$ :

$$
\begin{aligned}
& Q_{e}=\left(\overrightarrow{R_{e i}}, \overrightarrow{V_{e}}-\overrightarrow{V_{i}}\right)+\left(\overrightarrow{R_{e n}}, \overrightarrow{V_{e}}-\overrightarrow{V_{n}}\right)+\left(\overrightarrow{R_{T}}, \overrightarrow{V_{e}}-\overrightarrow{V_{n}}\right) \\
& \overrightarrow{R_{e i}}=-v_{e i} m_{e} N_{e}\left\{0.5\left(V_{e \|}-V_{i \|}\right) \vec{h}+\left(\overrightarrow{V_{e \perp}}-\overrightarrow{V_{i \perp}}\right)\right\} \\
& \overrightarrow{R_{e n}}=-v_{e n} m_{e} N_{e}\left\{0.5\left(V_{e \|}-V_{n \|}\right) \vec{h}+\left(\overrightarrow{V_{e \perp}}-\overrightarrow{V_{n \perp}}\right)\right\} \\
& \overrightarrow{R_{T}}=-0.7 N_{e}(\vec{h}, \nabla) T_{e}-\frac{3}{2} N_{e} \frac{v}{\Omega_{e}}\left[\vec{h}, \nabla T_{e}\right] .
\end{aligned}
$$

As a result

$$
Q_{e}=-\frac{\left(v_{e i}+v_{e n}\right) m_{e} N_{e}}{N_{e}^{2} e^{2}}\left\{0.5 j_{\|}^{2}+j_{\perp}^{2}+\frac{0.7 N_{e}^{2} e}{\mu_{0}}(\vec{h} \cdot \nabla \times \vec{B})(\vec{h}, \nabla) T_{e}\right\} ;
$$

here we neglect the diference between the ion and neutral velocities. The last term in the equation of energy balance to be kept describes the heat flux that is written as $\overrightarrow{q_{e}}=\overrightarrow{q_{u e}}+\overrightarrow{q_{T e}}$, with

$$
\begin{aligned}
\overrightarrow{q_{u e}} & =0.7 N_{e} T_{e}\left(V_{e \|}-V_{n \|}\right)+\frac{3}{2} N_{e} T_{e} \frac{v}{\Omega_{e}}\left[\vec{h}, \overrightarrow{V_{e \perp}}-\overrightarrow{V_{i \perp}}\right] \\
\overrightarrow{q_{e}} & =-3.16 \frac{N_{e} T_{e}}{m_{e}\left(v_{e n}+v_{e i}\right)} \nabla_{\|} T_{e}-4.66 \frac{N_{e} T_{e}\left(v_{e n}+v_{e i}\right)}{m_{e} \Omega_{e}^{2}} \nabla_{\perp} T_{e}-\frac{5}{2} \frac{N_{e} T_{e}}{m_{e} \Omega_{e}}\left[\vec{h}, \nabla_{\perp} T_{e}\right] .
\end{aligned}
$$

We shall begin by evaluating the energy supply provided by resistive dissipation that is determined by the first two terms in the right hand side of the equation of energy balance. The volumetric heating power of the electrons can be estimated as

$$
\frac{3}{2} n k_{B} \frac{d T_{e}}{d t} \sim j_{\|} E_{\|} \sim \frac{v_{e n}}{\mu_{0}} \frac{d^{2}}{L^{2}} \delta B^{2} \sim 10^{-4} \sim 10^{-3} \frac{W}{m^{3}} .
$$


In a stationary equilibrium, assuming that the macroscopic flow evacuates the power supplied by collisional resistive dissipation, one can estimate the temperature variation with altitude as

$$
\frac{d T_{e}}{d t}=V_{e z} \frac{\partial T_{e}}{\partial z}, \frac{\partial T_{e}}{\partial z} \sim \frac{j_{\|} E_{\|}}{\frac{3}{2} n k_{B} V_{e z}},
$$

where $V_{e z}$ is the characteristic macroscopic vertical velocity of electron (plasma) motion that can be evaluated to be of the order of the sound velocity. Then

$$
\frac{\partial T_{e}}{\partial z} \sim \frac{j_{\|} E_{\|}}{\frac{3}{2} n k_{B} V_{S}} \sim 0.01-0.1 \mathrm{eV} / \mathrm{km} .
$$

It is easy to see that the collisional heating of the electrons becomes rather efficient. Another possible evaluation of the temperature variation with altitude can be obtained by assuming a stationary static thermal equilibrium. Under such conditions the power supply would be balanced by any of several possible channels for energy losses. Assuming that the energy release is confined by the electron heat flux described by the term $\nabla q_{e}$ in Eq. (7) one can find

$$
\nabla \cdot \overrightarrow{q_{e}} \approx \frac{\partial}{\partial z} \kappa_{z}^{e} \frac{\partial T_{e}}{\partial z},
$$

where $\kappa_{z}$ is the electron thermal conductivity in the parallel direction. For our conditions when electron-neutral conditions are dominant in the parallel direction

$$
\kappa_{z}^{e} \approx \gamma \frac{n_{e} T_{e}}{m_{e} v_{e n}},
$$

where $\gamma$ is a coefficient of order unity (according to Braginskii [34]) for electron-ion collisions; when ions are singly ionized, it is equal to $\sim 3.8$. This leads to an estimate of the characteristic heat loss rate as

$$
\nabla \cdot \overrightarrow{q_{e}} \approx \frac{\partial}{\partial z} \gamma \frac{k_{B} n_{e} T_{e}}{m_{e} v_{e n}} \frac{\partial k_{B} T_{e}}{\partial z} \sim \frac{k_{B}^{2} n_{e} T_{e}^{2}}{m_{e} v_{e n} L_{T}^{2}},
$$

where $\left(T_{e} / L_{T}\right)$ is the characteristic variation of temperature with altitude. Under the physical conditions of the chromosphere this will lead to

$$
\left(\frac{T_{e}}{L_{T}}\right) \sim\left(\frac{j_{\|} E_{\|} m_{e} v_{e n}}{k_{B}^{2} n_{e}}\right)^{1 / 2} \sim 0.1-0.3 \mathrm{eV} / \mathrm{km} .
$$

Taking into account an exponential decrease of the plasma density, one can find that it can heat the plasma to the hydrogen ionization energy $(13.6 \mathrm{eV})$ on a characteristic distance of the order of a few hundred $\mathrm{km}$. It should be noted however that the thermal conductivity increases with the temperature and this can result in some flattening of the temperature profile.

Ion heating due to the perpendicular electric field component can be evaluated as

$$
\frac{d T_{i}}{d t} \sim\left(\overrightarrow{j_{\perp i}} \cdot\left\{\vec{E}+\left[\overrightarrow{V_{n}} \times \vec{B}\right]\right\}\right) \sim \frac{M_{i}}{m_{e}} \frac{\Omega_{i}^{2}}{v_{e n} v_{i n}} \frac{d T_{e}}{d t} .
$$


This estimate shows that the ion collisional heating can be several times smaller than or comparable to the electron heating.

\section{ON THE SIMILARITY OF PHYSICAL PROCESSES IN THE CHROMOSPHERE AND SEISMIC EFFECTS IN THE IONOSPHERE AFTER STRONG EARTHQUAKES}

The following material is based on the publication by Lognonne et al. [35]. Our model of electric-field generation and chromospheric electron heating can be compared with the observations of ionospheric perturbations after strong earthquakes, which involve physical mechanisms quite similar to those considered above. The perturbations resulting in the Earth's ionosphere are similar to those in the solar chromosphere. Ground-based seismic data are based on the detection of very small ground displacements: even at the most noisy frequency, $0.15 \mathrm{~Hz}$, associated with a global seismic noise generated by ocean waves, the amplitude of the ground displacement noise is in the range of $0.1-10 \mu \mathrm{m}$ and seismic waves with good signal-to-noise ratio can have amplitudes of a hundred to a thousand times higher.

The atmosphere is affected by waves, in a manner quite similar to the way in which photospheric flows enter the solar chromosphere. After an earthquake, seismic waves generate vertical and horizontal motions of the surface of the Earth. In some cases, tsunami or oceanic gravity waves are also generated and produce surface oscillations. By continuity of vertical displacement at the surface, the atmosphere is then forced to move with the same vertical velocity as the ground surface. The perturbation propagates upward as an atmospheric wave and produces pressure and temperature variations, and oscillations of the atmospheric layers. The seismic waves with the largest ground amplitude are the surface waves and especially the Rayleigh waves. These propagate along the Earth's surface, in the crust or upper mantle, with velocities ranging from 3 to $4 \mathrm{~km} / \mathrm{s}$. For large and superficial quakes, their displacement amplitude, even at an epicentral distance of $10^{4} \mathrm{~km}$, can reach several $\mathrm{mm}$ or even $\mathrm{cm}$. The propagation speed of the wave front being much larger than the sound speed in the atmosphere, the generated air waves propagate almost vertically from the surface location of the Rayleigh wave front. With their long periods $(T>10-20 \mathrm{~s})$, the infrasonic atmospheric waves are not attenuated by the atmospheric viscosity: they propagate with a constant kinetic energy and therefore, their amplitude grows exponentially as the inverse of the square root of density quite similar to waves in the chromosphere. As shown by Figure 6, the density decays by 10 orders of magnitude between the ground and $200 \mathrm{~km}$ altitude, and amplification factors of $10^{5}$ can therefore be encountered by these waves during their upward propagation. The main difference with the solar chromosphere is in characteristic scales: for the Earth the scale height $\mathrm{H} \approx 14 \mathrm{~km}$, while for the chromosphere it is $\sim 10 \times$ larger. On the other hand the efficiency of wave generation by the Rayleigh waves on the Earth's surface is much smaller, and their amplitudes are significantly weaker.

It is not only the neutral atmosphere that will oscillate following an earthquake. Collision processes produce an energy transfer to the ionospheric electrons and ions, 
which finally will result in generation of the electric currents and charge separation. For quakes of magnitude 7 or more, the ionosphere electrons oscillate with velocities of a few tens of $\mathrm{m} / \mathrm{s}$ and are displaced by a few hundred $\mathrm{m}$. These forced ionospheric waves, with horizontal speed imposed by the true solid Earth surface waves, are the target for remote-sensing observations of the surface waves.
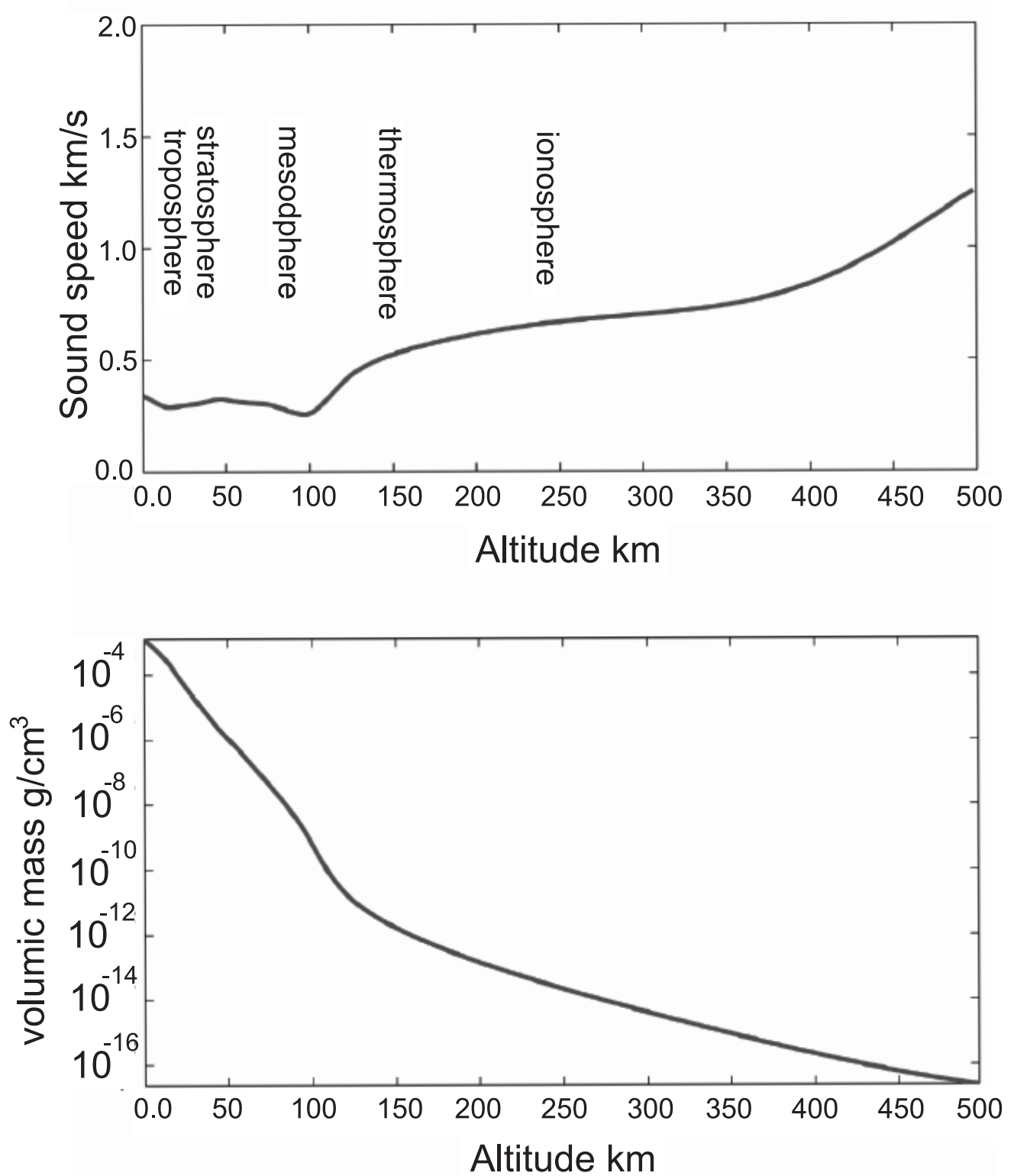

FIGURE 6. (Adapted from [35]). Density and sound speed profiles with altitude in the Earth's atmosphere. The ionosphere typically develops above $120 \mathrm{~km}$ altitude and the maximum of ionization is reached at $350-400 \mathrm{~km}$. Reproduced with permission, copyright (2007) European Physical Society.

The first observation of ionospheric surface waves were obtained after a very large Alaskan earthquake in 1964. At that time, the ionosphere was monitored for the purpose of nuclear explosion detection, and both the theories and the instruments necessary for 
the interpretation of the atmospheric gravity waves generated by megaton-scale atmospheric explosions had been developed [36], especially at the Seismological Laboratory of the California Institute of Technology. The pressure fluctuations generated by earthquakes are much smaller than those generated by typical gravity waves. The seismic source is indeed located in the solid earth and the coupling between the solid part and the atmosphere transfers only $10^{-4}$ to $10^{-5}$ of the energy [37]. Resonances are however found first near $3.7 \mathrm{mHz}$, where spheroidal modes have up to $0.05 \%$ of their energy in the atmosphere, and also near $4.4 \mathrm{mHz}$. These two maxima result from a wavelengthmatching of the Rayleigh waves with the mesospheric wave guide [37] and can lead to typical bi-chromatic seismic signals after volcanic atmospheric explosions [38]. Even for a magnitude 8 quake, the pressure fluctuations reach only a few microbars at the surface and must be recorded with complex measurement systems. The Earth atmosphere is however acting as a natural amplifier and leads to large signals at $150 \mathrm{~km}$ or more of altitude in the ionosphere, for earthquakes of magnitude 7 or larger.

The ionospheric oscillations can be remotely sounded by a Doppler sounder [39]. The principle of the Doppler sounder is to probe with electromagnetic waves in the range 1-15 MHz. The emitted wave reaching the plasma modifies its propagation as the electron density increases and will be fully reflected at a given altitude, where the plasma frequency (a function of local electron density) matches the radio wave frequency. If the reflecting layer is oscillating vertically, a Doppler effect is indeed observable in the reflected signal. The ground-based Doppler sounding can therefore follow the oscillations in ionospheric layers up to $350 \mathrm{~km}$ altitude, where the maximum ionization is reached. Modern instruments can detect vertical velocities of a few times $10 \mathrm{~cm} / \mathrm{s}$, enabling therefore the detection of all quakes with magnitude greater than about 7 (Fig. 7). Comparisons of these observations with modelling based on a system of equations, quite similar to those we obtained above for describing electric field generation, show very good agreement. Moreover the use of the GPS stations has resulted in detailed analysis of propagation of the perturbations of acoustic-gravity waves to large distances from the sources and the reconstruction of spatial pictures of the perturbation distributions.

\section{DISCUSSION}

The current generation mechanism that we propose here is strongly dependent upon four basic parameters: the velocity of the turbulent gas motions, the background magnetic field, the characteristic frequency of the spectrum of turbulent motions of the gas, and the characteristic spatial scale of velocity shear. The physical process of the generation of electric currents and magnetic fields is actually nothing other than the well-known turbulent-dynamo mechanism operating as a result of the the freezing of the magnetic field into the flow of the neutral gas.

The altitudes where this mechanism will operate are strongly dependent upon the local magnetic field strength: the stronger the field, the lower the altitude of electron magnetization. The efficiency of the electric current generation is proportional to the magnitude of the velocity shear. The characteristic spatial scales are proportional to the characteristic scales of this velocity shear, i.e. the flow vorticity. It is interesting 

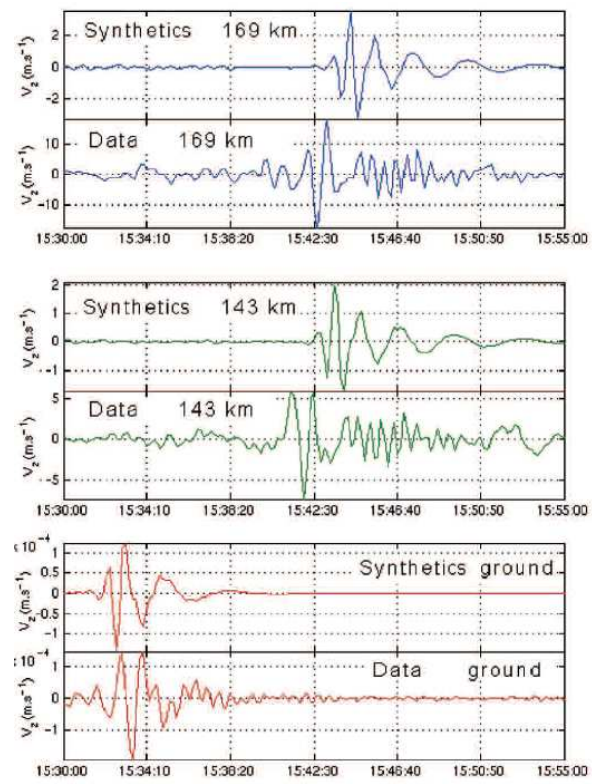

FIGURE 7. (Adapted from [35]). These signals correspond to the ionospheric vertical velocity. They are recorded by the Doppler sounder of CEA, at 143 and $169 \mathrm{~km}$ altitude, by using the reflection of two radio waves at 3.849 and $4.624 \mathrm{MHz}$ respectively. Data were recorded after a magnitude 8 earthquake in the South Indian Ocean, on June 18, 2000. The bottom trace corresponds to the data recorded by a seismometer. For all data, synthetic models have been computed for a spherically symmetric Earth, including both solid Earth and the atmosphere. Most of the differences at the ground are associated with the 3D structure of the Earth, which is not taken into account in the models. Differences in the amplitude of the ionospheric waves are probably due to a incorrect viscosity profile in the atmosphere, such data being badly known at high altitude. Data from CEA-DASE are reprinted from [39]. Reproduced with permission, copyright (2007) European Physical Society.

to note that in this formalism, magnetic fields and currents can be generated by both compressible and incompressible motions (see the first and second terms in Eq. (5)). Compressive motion tends to amplify or weaken the background magnetic field, while the rotational component effectively generates the perpendicular or helical components of the field. This means that compressional motions tend to generate transverse currents, while non-compressional motions can generate field-aligned currents as well.

Since density tends to decrease exponentially with height in the chromosphere, the characteristic velocity of chromospheric flows can increase by a factor of ten or more over their photospheric values. This gives rise to an estimate of the characteristic magnetic field generated by such motions readily becoming comparable to or even larger than the background field. Their relative magnitude is determined by the ratio of the characteristic velocity of neutral motions at the altitude of electron magnetization to the characteristic parameter $\omega H$. One can see that for characteristic frequencies of the order of $10^{-2}-10^{-3} \mathrm{~s}^{-1}$, and characteristic heights of the order of $140 \mathrm{~km}$, the field generation becomes quite efficient already for velocities of the order of tens of $\mathrm{km} / \mathrm{s}$. The helical magnetic field component can become larger than the background field. A similar estimate shows that amplification/weakening of the background magnetic field component can be evaluated to be of the order of $b / B_{0} \sim \delta n / n$. Under such conditions, 
we show that the electron heating can become rather efficient due to the collisional resistive dissipation of the electric current. These processes take place in the regions where the magnetic field vorticity increases.

It is worth noting that the efficiency of the electron and ion heating can become substantially more important than that due to the collisional resistive dissipation described above. The electric current and magnetic field generation we have discussed takes place at the chromospheric footpoints of flux tubes that may extend into the corona. The direction of the generated field is determined by the characteristics of the gas velocity shear and can lead to the formation of the magnetic field configurations that can become unstable, or can form new structures by interaction with neighbouring fields with different orientations. The increase of the perpendicular component of the magnetic field can result in a kink instability if this component satisfies the Kruskal-Shafranov condtion $\delta B / B>l / R$. Here $l$ is the characteristic scale of the cross section of the tube and $R$ is its characteristic length. Under such conditions the tube cannot keep its configuration intact; it will become unstable and then different kinds of perturbations will result in its reconfiguration. Other types of emerging configurations with magnetic field inversions will result in local reconnection. The detailed study of these effects lies beyond the scope of our paper and will be carried out elsewhere.

The result of the heating would be a rapid increase of the degree of ionization of the gas. It is worth remembering that our whole description is valid only when the degree of ionization is low. This rapid ionization process can lead, according to most radiativeconvective models [e.g., 40] to an efficient decrease of the radiative cooling rate of the gas. Thus the generation of electric currents results not only in the plasma heating itself, but also causes a decrease of the cooling process that will then strengthen the effect of radiative heating. We cannot make any quantitative estimate of these effects, but they may be adressed in future simulations.

The coupling of acoustic-type waves and MHD waves can also be treated in the MHD approximation [e.g., 41]. The major difference in our approach here is that the neutral collisions reduce the feedback effect on the motion of the gas resulting from the newly generated magnetic field. This produces a more efficient transfer of energy from gas motions to current generation and magnetic field amplification.

It seems possible to incorporate certain aspects of this formalism into a system of conservation equations similar to those used in [42]. In that work, the resistive MHD equations were solved numerically within a computational domain that includes both a turbulent model convection zone and corona. We are currently updating this system of equations to incorporate additional physics, and are planning to perform a comparison between a standard resistive radiative-MHD model and our new results. We hope to report on the results of this study in the near future.

\section{CONCLUSION}

We have presented an analysis of the effect of electric current and associated magnetic fields generation in a "chromospheric dynamo layer" where electrons become magnetized while ions remain collisionally coupled to the neutrals. We have shown that electric currents and magnetic fields can be generated very efficiently due to turbulent motions 
of the neutral gas. The efficiency of this physical process is proportional to the characteristic velocity shear of the gas turbulent motions and inversely proportional to their characteristic frequency. We also found that the magnetic fields thus generated can be comparable to or even larger than the background magnetic field for motions having characteristic scales of the order of several hundred $\mathrm{km}$ and characteristic time scales of the order of several minutes. This can produce a substantial restructuring of magnetic field configurations and an opportunity to create multiple sites of reconnection. It also results in an efficient increase of collisional resistive heating of electrons and ions, and hence the rapid ionization of the gas, thus unstably altering its thermal equilibrium.

The similarity of parameters in the Earth's ionosphere and the Sun's chromosphere provides an opportunity to refer to observations of ionospheric perturbations, after strong earthquakes, for evaluation of the effect of amplification of sound-like perturbations in the solar chromosphere. Simple reasoning based on scaling shows that the effects described in [28] can be responsible for chromospheric heating and ionization.

\section{ACKNOWLEDGMENTS}

The authors are grateful to CNES for financial support in the frame of CNES "Solar Orbiter Research grant". H. H. thanks NASA for support under NAG5-12878. V. K. acknowledges very useful discussions with M. Ruderman, M. Velli and A. Artemyev.

\section{REFERENCES}

1. J. A. Klimchuk, Solar Phys. 234, 41-77 (2006), arXiv : astro-ph/ 0511841.

2. R. W. Walsh, and J. Ireland, Astron. Astrophys. Rev. 12, 1-41 (2003).

3. E. N. Parker, Astrophys. J. 330, 474-479 (1988).

4. R. P. Lin, R. A. Schwartz, S. R. Kane, R. M. Pelling, and K. C. Hurley, Astrophys. J. 283, 421-425 (1984).

5. P. J. Cargill, Astrophys. J. 422, 381-393 (1994).

6. Y. Sakamoto, S. Tsuneta, and G. Vekstein, Astrophys. J. 689, 1421-1432 (2008).

7. G. Vekstein, Astron. Astrophys. 499, L5-L8 (2009).

8. T. Shimizu, S. Tsuneta, L. W. Acton, J. R. Lemen, Y. Ogawara, and Y. Uchida, Astrophys. J. 422, 906-911 (1994).

9. S. Krucker, A. O. Benz, T. S. Bastian, and L. W. Acton, Astrophys. J. 488, 499-+ (1997).

10. R. A. Harrison, Solar Phys. 175, 467-485 (1997).

11. S. Koutchmy, H. Hara, Y. Suematsu, and K. Reardon, Astron. Astrophys. 320, L33-L36 (1997).

12. D. Berghmans, F. Clette, and D. Moses, Astron. Astrophys. 336, 1039-1055 (1998).

13. A. O. Benz, and S. Krucker, Solar Phys. 182, 349-363 (1998).

14. H. S. Hudson, Solar Phys. 133, 357-369 (1991).

15. A. F. Rappazzo, M. Velli, G. Einaudi, and R. B. Dahlburg, Astrophys. J. 677, 1348-1366 (2008), 0709.3687.

16. B. De Pontieu, S. McIntosh, V. H. Hansteen, M. Carlsson, C. J. Schrijver, T. D. Tarbell, A. M. Title, R. A. Shine, Y. Suematsu, S. Tsuneta, Y. Katsukawa, K. Ichimoto, T. Shimizu, and S. Nagata, Pub. Astron. Soc. Japan 59, 655-+ (2007), 0710.2934.

17. Y. Lin, O. Engvold, L. Rouppe van der Voort, J. E. Wiik, and T. E. Berger, Solar Phys. 226, 239-254 (2005).

18. T. J. Okamoto, S. Tsuneta, B. W. Lites, M. Kubo, T. Yokoyama, T. E. Berger, K. Ichimoto, Y. Katsukawa, S. Nagata, K. Shibata, T. Shimizu, R. A. Shine, Y. Suematsu, T. D. Tarbell, and A. M. Title, Astrophys. J. 697, 913-922 (2009), 0904.0007. 
19. K. Shibata, T. Nakamura, T. Matsumoto, K. Otsuji, T. J. Okamoto, N. Nishizuka, T. Kawate, H. Watanabe, S. Nagata, S. UeNo, R. Kitai, S. Nozawa, S. Tsuneta, Y. Suematsu, K. Ichimoto, T. Shimizu, Y. Katsukawa, T. D. Tarbell, T. E. Berger, B. W. Lites, R. A. Shine, and A. M. Title, Science 318, 1591- (2007), 0810.3974.

20. D. M. Rust, "Chromospheric Explosions and Satellite Sunspots," in Structure and Development of Solar Active Regions, edited by K. O. Kiepenheuer, 1968, vol. 35 of IAU Symposium, pp. 77-+.

21. W. P. Abbett, Z. Mikić, J. A. Linker, J. M. McTiernan, T. Magara, and G. H. Fisher, Journal of Atmospheric and Solar-Terrestrial Physics 66, 1257-1270 (2004).

22. W. P. Abbett, Astrophys. J. 665, 1469-1488 (2007).

23. B. De Pontieu, P. C. H. Martens, and H. S. Hudson, Astrophys. J. 558, 859-871 (2001).

24. J. M. Fontenla, W. Curdt, M. Haberreiter, J. Harder, and H. Tian, Astrophys. J. 707, 482-502 (2009).

25. O. Steiner, "Photospheric processes and magnetic flux tubes," in Kodai School on Solar Physics, edited by S. S. Hasan \& D. Banerjee, 2007, vol. 919 of American Institute of Physics Conference Series, pp. 74-121.

26. J. W. Harvey, D. Branston, C. J. Henney, C. U. Keller, and SOLIS and GONG Teams, Astrophys. J. Lett. 659, L177-L180 (2007), arXiv:astro-ph/ 0702415.

27. B. W. Lites, M. Kubo, H. Socas-Navarro, T. Berger, Z. Frank, R. Shine, T. Tarbell, A. Title, K. Ichimoto, Y. Katsukawa, S. Tsuneta, Y. Suematsu, T. Shimizu, and S. Nagata, Astrophys. J. 672, 1237-1253 (2008).

28. V. Krasnoselskikh, G. Vekstein, H. S. Hudson, S. D. Bale, and W. P. Abbett, Astrophys. J. 724, 1542-1550 (2010), 1011. 5834.

29. J. Klostermeyer, JASTP 31, 25-45 (1969).

30. J. Klostermeyer, Ann. Geophys. 25, 547-550 (1969).

31. R. F. Stein, and A. Nordlund, Astrophys. J. 499, 914 (1998).

32. T. Straus, B. Fleck, S. M. Jefferies, S. W. McIntosh, G. Severino, M. Steffen, and T. D. Tarbell, "On the Role of Acoustic-Gravity Waves in the Energetics of the Solar Atmosphere," in The Second Hinode Science Meeting: Beyond Discovery-Toward Understanding, edited by B. Lites, M. Cheung, T. Magara, J. Mariska, \& K. Reeves, 2009, vol. 415 of Astronomical Society of the Pacific Conference Series, p. 95, 1003.3773.

33. A. Fossum, and M. Carlsson, Astrophys. J. 646, 579-592 (2006).

34. S. I. Braginskii, Reviews of Plasma Physics 1, 205 (1965).

35. P. Lognonne, G. Raphael, C. Francois, O. Giovanni, K. Alam, and A. Juliette, EurophySicsnews 37, 11-14 (2006).

36. D. G. Harkrider, J. Geophys. Res. 69, 5295-5321 (1964).

37. P. Lognonné, E. Clévédé, and H. Kanamori, Geophysical Journal International 135, 388-406 (1998).

38. H. Kanamori, and J. Mori, Geophys. Res. Lett. 19, 721-724 (1992).

39. J. Artru, T. Farges, and P. Lognonné, Geophysical Journal International 158, 1067-1077 (2004).

40. R. F. Stein, and A. Nordlund, Astrophys. J. 499, 914-+ (1998).

41. J. V. Hollweg, S. Jackson, and D. Galloway, Solar Phys. 75, 35-61 (1982).

42. W. P. Abbett, Astrophys. J. 665, 1469-1488 (2007). 